\title{
The Relationship Between Cognitive and Affective Empathy and Human Mating Strategies
}

\author{
Rafael Wlodarski ${ }^{1}$
}

Published online: 12 August 2015

(C) Springer International Publishing 2015

\begin{abstract}
Humans display a wide variety of mating strategies, ranging from short-term (promiscuous) strategies to long-term (monogamous) strategies. It has been previously suggested that certain personality factors, including individual differences in empathy, may be related to the pursuit of differing mating strategies. The aim of this cross-sectional study was to examine the relationships between different forms of empathy and the inclination towards pursuing a short-term mating strategy, as indexed by sociosexuality. It was found that cognitive empathy abilities were positively associated with higher levels of sociosexuality, though much more so for individuals who were also high in affective empathy. When examining the various sub-components that make up sociosexuality, it was found this effect may be driven by the desire aspect of sociosexuality. It appears, therefore, that the ability to cognitively understand the emotional state of other individuals is related to promiscuous mating strategies, especially when combined with the ability to intuitively feel the emotions of the other individual.
\end{abstract}

Keywords Empathy · Mating strategies · Sociosexuality · Reading the mind in the eyes $\cdot$ Empathy quotient

\section{Introduction}

Humans are an inherently social species, living in large groups and having to navigate various forms of social interaction on a

Rafael Wlodarski

rafael.wlodarski@psy.ox.ac.uk

1 Department of Experimental Psychology, University of Oxford, South Parks Road, Oxford OX1 3UD, UK daily basis (Shultz and Dunbar 2007). One of the key abilities required to successfully engage with other individuals involves having some form of understanding of other's mental and emotional states, so as to better coordinate behaviors or avoid confrontations. A capacity which is thought to be crucial to dealing with the complexities of the social environment is empathy, which is generally described as the ability to intuit and/or evaluate the emotional mental state of another individual (Singer 2006).

Empathy, however, is a rather complicated construct, with disagreements arising as to its exact scope and meaning. While some authors would argue for a multi-faceted description incorporating up to eight different components (Batson 2009), empathy is most commonly divided into two distinct but related constructs: an affective component, which involves the elicitation of an intuitive and homologous emotional response in an observer (i.e., "I feel what you feel"), and a cognitive component, which involves the intellectual understanding and recognition of another's emotional mental state (i.e., "I understand what you feel") (Blair 2005; Henry et al. 2008; Shamay-Tsoory et al. 2009). The cognitive element of empathy has some conceptual overlap with the broader concept of "Theory of Mind" or mentalizing, that is the general ability to ascertain the mental state of another individual (Frith et al. 1991; Premack and Woodruff 1978). Because of this, it is sometimes also referred to as affective Theory of Mind, although it is still considered a distinct ability from mentalizing due to its exclusive focus on emotional content. Research utilizing both lesion studies as well as fMRI brain imaging techniques has shown that that these two forms of empathy are often independent, exhibiting both behavioral and neurological double disassociations (Saxe 2006; Shamay-Tsoory et al. 2009; Singer 2006; Walter 2012).

Empathy is important to the formation and maintenance of various types of social relationships, including mother-infant 
bonds, romantic relationships, and friendships, and is influential in the development of prosocial behaviors (Roberts and Strayer 2013). Both cognitive and affective forms of empathy are thought to be essential for successful social interaction to take place, as illustrated by psychiatric disorders which have been associated with severe deficits in either one of these forms of empathy. It has been suggested, for example, that individuals with autism spectrum disorders have difficulties with cognitive empathy abilities, though not affective empathy abilities, and thus find it difficult to ascertain and describe the mental states of other individuals, including their emotional state (Baron-Cohen et al. 1985; Blair 2005). In contrast, individuals with pathological psychopathy typically show limited affective empathy abilities, exemplified in an inability to feel analogous emotions with target others, while retaining the ability to discern and describe such emotions (Blair 2005; Hare 1980). The presence of either of these conditions is typically related to severe impairments in the formation and maintenance of different types of social relationships. Although such pathologies illustrate the deleterious social effects of severe impairments in empathizing abilities, there also exists considerable variation in affective and cognitive empathy abilities within non-pathological populations, variation which may explain differences in social function.

Differences in empathy abilities have been particularly closely studied in the area of romantic (mating) relationships, where the ability to empathize appears to play an important role in long-term relationship maintenance. A large body of past research has found, for example, that empathy abilities are positively related to relationship satisfaction and reduced partner conflict (Boettcher 1978; Busby and Gardner 2008; Cramer and Jowett 2010; Davis and Oathout 1987; Levesque et al. 2014; Perrone-McGovern et al. 2014). Research examining brain activations using fMRI, meanwhile, finds that activations in regions associated with empathy (including the anterior insula) are stronger among individuals in long-term relationships which had greater levels of marital satisfaction (Acevedo and Aron 2012). Much of this research, however, has typically used cross-sectional designs, meaning that conclusions about causality are difficult to make. One intervention study, however, found that empathy training sessions had a direct positive effect on follow-up relationship satisfaction (Long et al. 1999). It is likely that being able to empathize and better understand a partner's emotional and mental state may facilitate the coordination of motivations and behaviors between two individuals, while also aiding in the monitoring of the state and health of the relationship (Wlodarski and Dunbar 2014).

This tendency to form long-term sexual relationships appears to be a universal human trait (Jankowiak and Fischer 1992), allowing for the creation of mating pair bonds which facilitate coordination of bi-parental behaviors and resources, which in turn improve chances of offspring survival (CluttonBrock 1989; Geary 2000). However, humans are known to also pursue a variety of other mating strategies (Buss and Schmitt 1993), including relatively "short-term" mating strategies. The degree to which an individual favors a short-term mating strategy, involving a preference for promiscuous mating with multiple short-term partners, is referred to as their sociosexual orientation (Penke and Asendorpf 2008; Simpson et al. 1991). The greatest interpersonal differences in mating strategy exist between the sexes: parental investment theory suggests that the sex undertaking the greater level of minimal parental investment (in humans, the female) will be more selective and less likely to pursue short-term mating strategies (Andersson 1994; Trivers 1972). This has been confirmed in humans, with males in every known culture found to be more likely to pursue short-term mating strategies than females (Lippa 2009; Schmitt 2005). However, considerable variation in mating strategy has also been found to exist within each of the sexes (e.g., Bailey et al. 2000; Gangestad and Simpson 2000; Wlodarski et al. 2015). Such mating strategy differences, moreover, seem to be both relatively stable over the lifetime and to have an underlying genetic component (Bailey et al. 2000; Bleske-Rechek et al. 2009).

Individuals pursuing short-term mating strategies tend to focus on different mate-choice criteria, displaying greater focus on fecundity and genetic "fitness" in potential mates versus resource acquisition potential or traits related to "good parenting" (Gangestad and Simpson 2000). Past research suggests that these individuals may also be more adept at detecting mate cues which are relevant to the pursuit of short-term strategies. For example, men pursuing short-term mating strategies show greater sensitivity to female facial cues of fertility (Sacco et al. 2009), while females interested in short-term mating show greater attractiveness to "genetically fit" faces (Waynforth et al. 2005) and better episodic memory for fitness-related cues in males (Smith et al. 2013). Across both sexes, high sociosexuality (short-term mating focused) individuals show reduced levels of sexual disgust (but not moral or pathogen disgust) (Al-Shawaf et al. 2014), greater perceptual bias of sexual intent in potential mating partners (Howell et al. 2012), and more behaviors aimed at limiting emotional engagement (Haselton and Buss 2001; Jonason and Buss 2012). Such individuals also appear to share certain personality components-including higher extraversion and lower agreeableness and conscientiousness (Schmitt 2008).

Short-term mating strategies have been linked to another cluster of personality factors known as the Dark Triad - which incorporates non-clinical psychopathy, narcissism, and Machiavellianism (Paulhus and Williams 2002). These three personality schemas share some commonalities-including reduced levels of empathy independently associated with narcissism (Watson et al. 1984), psychopathy (Blair 2006), and Machiavellianism (Barnett and Thompson 1985). It has been 
suggested that low empathy may assist in the establishment of "manipulative relationships" typically associated with the Dark Triad by reducing an individual's ability to empathize with any pain or anguish caused by such exploitative behavior. Closer inspection of different forms of empathy, however, suggests that it may be a combination of low affective empathy (found in psychopathy) and high cognitive empathy (found in narcissism) which may be responsible for high levels of social manipulation (Wai and Tiliopoulos 2012). Wai and Tiliopoulos conclude that "high dark triad individuals exhibit substantial desensitisation towards the negative emotions of others, which, augmented by an intact cognitive empathy, likely aids their callous and manipulative nature" ( $p$. 798).

Whether such a disassociation of empathy is directly related to short-term mating strategies among Dark Triad populations is at this point uncertain. In non-pathological populations, it is possible that reduced levels of affective empathy may aid in the pursuit of short-term mating strategies by reducing an individual's ability to reciprocate any feelings of romantic attachment which might arise in a mating partner. This trait would be analogous to other behaviors aimed at limiting emotional engagement previously found to be more common among short-term mating focused individuals (e.g., Haselton and Buss 2001; Jonason and Buss 2012). While this type of empathy is likely to aid in the formation and maintenance of long-term relationships, it could potentially serve as a hindrance to individuals pursuing a short-term mating strategy, as individuals would have to trade-off alternate mating opportunities if they were continually being engaged in prolonged pair-bonded relationships (Buss and Schmitt 1993). Poor affective empathy abilities may also adversely affect long-term relationship survival, and thus individuals with such poor skills may be limited to engaging in short-term mating relationships. Improved cognitive empathy skills, on the other hand, may prove useful in pursuing short-term mating goals, allowing individuals to quickly and efficiently cogitate on the contents of others' mind states, thus facilitating cohesive social interaction and the rapid establishment of mating relationships. In such encounters, both time and background information may be limited, and rapid and accurate mentalizing abilities may be useful if the goal is to initiate a short-term mating encounter. A better understanding of the mind states of others may also help promiscuous individuals find and select likeminded partners. Of course, such cognitive empathy abilities would also be useful in the establishment and maintenance of various other forms of social relationships, including longterm mating relationships, and are thus not likely to be exclusively related to short-term mating.

Although low levels of general empathy have been previously indirectly associated with short-term mating orientation, and high levels of general empathy have been associated with successful long-term relationship maintenance, the exact relationship between cognitive and affective empathy and mating strategies has yet to be examined. The purpose of this exploratory study, therefore, was to investigate the association between individual variation in levels of cognitive and affective empathy and sociosexuality (or proclivity towards a shortterm mating strategy). Based on tangential previous research in this area, it was predicted that the cognitive component of empathy would likely be positively related to short-term mating strategies, while the affective component would either not have any association, or would be negatively associated, with short-term mating.

\section{Method}

An online questionnaire was distributed to participants based in the USA using the Mechanical Turk micro-task crowdsourcing platform. Participants were over 18 years old, were informed that their responses were voluntary and anonymous, provided informed consent, and were remunerated a standard Mechanical Turk payment amount for their time. The study was approved by the Oxford University's Research Ethics Committee (CUREC).

In total, 250 participants completed the survey; however, 17 participants were excluded a priori as the time taken to complete the survey $(<6 \mathrm{~min})$ was deemed to be insufficient to provide reliable answers. Of the remaining 233 participants, of whom 134 were male and 99 were female, participants ranged in age from 18 to $70(M=36.4, \mathrm{SD}=11.8)$, with $67 \%$ of participants stating that they were in a relationship at the time of the survey. As part of the survey, participants were asked to complete a short battery of standardized tests.

To assess cognitive empathy abilities, participants completed the "Reading the Mind in the Eyes" task (RTM: BaronCohen et al. 2001; Baron-Cohen et al. 1997), where they were presented with a series of 36 black and white photographs of cropped male and female eyes displaying a wide variety of emotions and asked to ascertain which emotion was being felt by each target set of eyes (choosing from among four possible options). A total empathy (RTM) score was attained by summing the number of correct answers. This task involves attributing relevant emotional mental states to others based on limited visual information and has been shown to detect subtle individual differences in social sensitivity in healthy populations, with good reliability (Baron-Cohen et al. 2001) and validity (performance on the task correlates with brain regions previously associated with cognitive empathy) (for a review, see Schurz et al. 2014). This test is often used to assess cognitive aspects of empathy, wherein individuals must successfully theorize about the emotional mental states of others, without necessarily having those feelings induced in themselves (see Henry et al. 2008; Lawrence et al. 2004; van Honk et al. 2011). 
To assess general empathy abilities, including an affective empathy component, participants completed the 22item self-report Empathizing Quotient (EQ), a standardized self-report scale assessing one's ability to empathize in various social situations. This scale includes questions such as "I find it easy to put myself in somebody else's shoes" and "I can tune into how someone else feels rapidly and intuitively," with responses collected on 4-point Likert-type scales ranging from "strongly disagree" to strongly agree" (Wakabayashi et al. 2006). Responses were reverse-scored where necessary-responses of "agree" were scored as 1 point and "strongly agree" as 2 points. These were totaled to create the affective empathy (EQ) score (Cronbach's $\alpha=.92$ ). The EQ is often used to assess empathy abilities (e.g., Wai and Tiliopoulos 2012), and although it assesses "general" empathy abilities, according to factor analyses, it is particularly sensitive to the affective component of empathy (Muncer and Ling 2006).

To measure participants' preference for short-term mating strategies, participants completed the 9-item Sociosexual Orientation Inventory-Revised (SOI-R), which assesses an individual's behaviors, attitudes, and desires (with three items evaluating each) as they relate to non-committal sex (Penke and Asendorpf 2008; Simpson et al. 1991). Sample questions included "Sex without love is OK" and "How often do you have fantasies about having sex with someone you are not in a committed romantic relationship with?"; responses to all items were collected on various 9-point Likert-type scales (i.e., $1=$ "strongly disagree," 9="strongly agree"; $1=$ ="never," $9=$ "at least once a day"). Participant responses were reversescored where necessary and averaged for all items to create a total sociosexuality (SOI) score $(\alpha=.87)$, with the three items comprising each of the sub-scales also averaged to create behavior sociosexuality $(\alpha=.75)$, attitude sociosexuality $(\alpha=.90)$, and desire sociosexuality $(\alpha=.91)$ sub-scores.

\section{Results}

As an initial exploration of the data, correlations between participant sex, cognitive empathy (RTM) $(M=25.8, \mathrm{SD}=4.8)$, affective empathy (EQ) $(M=22.1, \mathrm{SD}=9.3)$, and sociosexuality $(M=3.9, \mathrm{SD}=1.8)$ were carried out (see Table 1). The data suggested that sex was related to affective empathy (EQ), with females $(M=24.1, \mathrm{SD}=8.8)$ having higher overall scores than males $(M=20.1, \mathrm{SD}=9.4 ; t(229)=$ $2.81, p=.005, d=.38)$; sex was also related to sociosexuality, with males $(M=4.5, \mathrm{SD}=1.7)$ scoring higher on sociosexuality than females $(M=3.0, \mathrm{SD}=1.4 ; t(231)=6.79$, $p<.001, d=.89$ ).

To examine the relationship between sex and the two types of empathy and sociosexuality simultaneously and in more detail, including any possible interactions between these variables, a multiple regression analysis was run. In this regression, sex was coded as a binary variable (male $=0$ and female $=1$ ), the cognitive empathy (RTM) and affective empathy (EQ) variables were firstly standardized and then entered as main regressor, the product of standardized cognitive empathy $(\mathrm{RTM}) \times$ affective empathy $(\mathrm{EQ})$ was entered as an interaction term, and the product of sex $\times$ cognitive empathy (RTM) and sex $\times$ affective empathy (EQ) was entered as further interaction terms. The results of this analysis can be seen in Table 2 . The same analysis was also run with age added as an extra predictor; however, the change in $r^{2}\left(\Delta r^{2}=0\right)$ was not significant, and thus this variable was not included in the final model.

This regression analysis shows, firstly, that males have higher sociosexuality than females and that cognitive empathy (RTM) has a relationship with sociosexuality which is mediated by affective empathy (EQ). Figure 1 illustrates this interaction effect by charting four sample points from the regression equation (RTM score $\pm 1 \mathrm{SD}$ and EQ score $\pm 1 \mathrm{SD}$ ), thus demonstrating how the effect of low cognitive empathy (RTM) and high cognitive empathy (RTM) scores on sociosexuality is mediated by whether an individual also has a low affective empathy (EQ) or a high affective empathy (EQ) score. It appears that with individuals who have low affective empathy, differences in cognitive empathy have no relationship with sociosexuality, whereas among individuals with high affective empathy, higher cognitive empathy scores have an increasingly positive relationship with sociosexuality scores.

In order to explore the relationship between sex and the two types of empathy and the different sub-components of sociosexuality (behavior, attitudes, and desires), three separate
Table 1 Correlation matrix between sex, cognitive empathy (RTM), affective empathy (EQ), and sociosexuality

\begin{tabular}{llllll}
\hline & Mean (SD) & Sex & $\begin{array}{l}\text { Cognitive } \\
\text { Empathy (RTM) }\end{array}$ & $\begin{array}{l}\text { Affective } \\
\text { Empathy (EQ) }\end{array}$ & Sociosexuality \\
\hline Sex & & 1 & .065 & $.182^{*}$ & $-.408^{*}$ \\
Cognitive empathy (RTM) & $25.8(4.8)$ & & 1 & -.028 & .096 \\
Affective empathy (EQ) & $22.1(9.3)$ & & & 1 & -.013 \\
Sociosexuality & $3.9(1.7)$ & & & & 1 \\
\hline
\end{tabular}

For sex, male $=0$ and female $=1$, Pearson's $r$ correlations

${ }^{*} p<.01$ (two-tailed), level of significance 
Table 2 Regression analysis results of sex, cognitive empathy (RTM), affective empathy (EQ), and their interactions and sociosexuality score

\begin{tabular}{llllll}
\hline & $\beta$ & SE & $S \beta$ & $t$ & $p$ \\
\hline Intercept & 4.490 & .141 & & 31.83 & .001 \\
Sex & $\mathbf{- 1 . 4 8 4}$ & $\mathbf{. 2 1 8}$ & $\mathbf{- . 4 1 6}$ & $\mathbf{- 6 . 7 8}$ & $\mathbf{. 0 0 1}$ \\
Cognitive empathy & $\mathbf{. 3 0 2}$ & $\mathbf{. 1 4 1}$ & $\mathbf{. 1 7 1}$ & $\mathbf{2 . 1 5}$ & $\mathbf{. 0 3 3}$ \\
Affective empathy & .017 & .138 & .009 & 0.12 & .905 \\
Cognitive empathy $\times$ affective empathy & $\mathbf{. 2 5 0}$ & $\mathbf{. 1 2 3}$ & $\mathbf{. 1 2 7}$ & $\mathbf{2 . 0 4}$ & $\mathbf{. 0 4 2}$ \\
Sex $\times$ cognitive empathy & -.113 & .223 & -.040 & -0.51 & .613 \\
Sex $\times$ affective empathy & .190 & .222 & .067 & 0.86 & .393 \\
\hline
\end{tabular}

Bold indicates significance at $p>.05$

$r^{2}=.197$; for sex, male $=0$ and female $=1$ regression analyses were run on each of the sociosexuality sub-scale scores (Table 3).

These regression analyses show that there were no significant relationships between sex or either form of empathy and the behavioral sub-component of sociosexuality, that only sex was related to differences in the attitude sub-component, and that only sex was related to the desire sub-component, though the interaction term of cognitive empathy $\times$ affective empathy was close to being significantly related to the desired subcomponent $(p=.057)$.

\section{Discussion}

This study aimed to explore the relationship between the two components of empathy and individual variation in short-term mating strategy, as indexed by sociosexual orientation. It was found, firstly, that males tended to pursue short-term strategies more than females in nearly all cases. Secondly, it was found that cognitive empathy abilities (as assessed by the reading the Mind in the Eyes task) were positively associated with higher levels of sociosexuality, though much more so for individuals who were also high in general (affective) empathy (as assessed by the Empathy Quotient, EQ). When examining the various sub-components that make up sociosexuality, it was found this

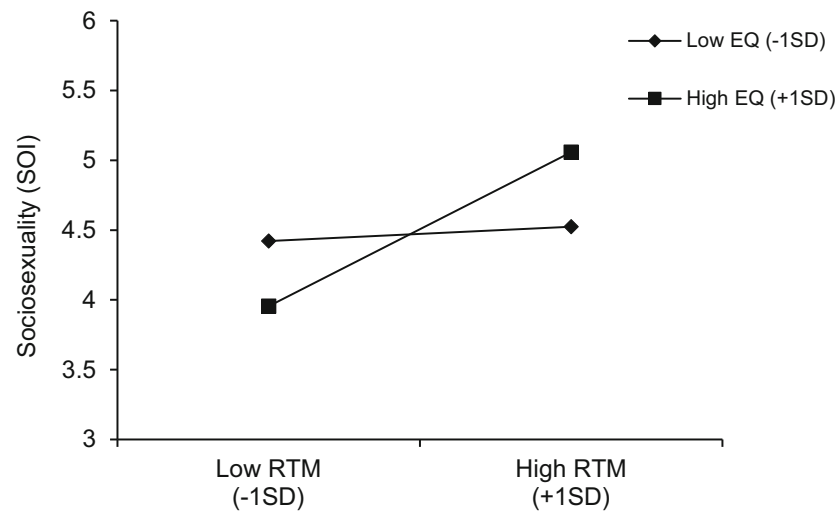

Fig. 1 Illustrative example of the interaction effect of cognitive empathy (RTM) and affective empathy (EQ) on sociosexuality scores effect may be driven by a similar trend in cognitive and affective empathy when it came to the desire component of sexuality.

Empathy abilities are useful in the initiation and maintenance of various social relationships: the ability to assess and monitor another's emotional mind state allows an understanding of their point of view, the formulation of an appropriate response, and the eventual establishment of rapport and a social bond with that individual (Roberts and Strayer 2013). This is likely the reason why pathological deficits in empathy abilities often co-vary with forms of impaired social function (e.g., autism or psychopathy: Baron-Cohen et al. 1985; Hare 1980). Empathy is likely equally important in the domain of mating relationships - with past research showing that high levels of general empathy are associated with the successful maintenance of long-term mating relationships (e.g., Boettcher 1978). This study is the first attempt to examine the relationship between various empathy traits and mating strategies, in particular the preferential pursuit of short-term mating strategies.

That sex differences were found in preferences for shortterm mating, with males scoring higher than females, is not surprising in light of a vast body of cross-cultural research (Lippa 2009; Schmitt 2005). Most interestingly, this study found that both cognitive empathy abilities and general (affective) empathy abilities interacted to show a positive relationship with short-term mating strategies. The results suggest that the among individuals with higher affective empathy, cognitive empathy was strongly positively related to shortterm mating strategy, while among individuals with low affective empathy, cognitive empathy had little to no association with mating strategies. The fact that cognitive empathy is related to short-term mating is congruent with previous research and theory - the ability to quickly and accurately assess another individual's (emotional) mind state may prove useful in the pursuit of short-term mating goals, aiding rapid initiation of intimate relationships by appropriately responding to another's mood state. This kind of ability may assist in the engineering of manipulative short-term mating relationships with individuals who might otherwise not be interested in such relationships. Alternatively, better assessment of other's mind states may also assist promiscuous individuals 
Table 3 Regression analysis results of sex, cognitive empathy (RTM), affective empathy (EQ), and their interactions and behavior, attitude, and desire sociosexuality sub-scores

\begin{tabular}{|c|c|c|c|c|c|}
\hline & $\beta$ & SE & $S \beta$ & $t$ & $p$ \\
\hline \multicolumn{6}{|l|}{ Behavior sociosexuality } \\
\hline Intercept & 2.891 & .145 & & 19.87 & .001 \\
\hline Sex & -0.272 & .225 & -.081 & -1.21 & .229 \\
\hline Cognitive empathy & 0.147 & .145 & .088 & 1.01 & .312 \\
\hline Affective empathy & 0.231 & .143 & .139 & 1.62 & .107 \\
\hline Cognitive empathy $\times$ affective empathy & 0.168 & .127 & .090 & 1.33 & .185 \\
\hline Sex $\times$ cognitive empathy & -0.122 & .230 & -.046 & -0.53 & .597 \\
\hline Sex $\times$ affective empathy & 0.134 & .229 & .050 & 0.58 & .560 \\
\hline \multicolumn{6}{|l|}{ Attitude sociosexuality } \\
\hline Intercept & 5.989 & .226 & & 26.46 & .001 \\
\hline Sex & -2.030 & .350 & -.363 & $-\mathbf{5 . 8 0}$ & .001 \\
\hline Cognitive empathy & 0.385 & .226 & .139 & 1.71 & .089 \\
\hline Affective empathy & -0.176 & .222 & -.064 & -0.79 & .429 \\
\hline Cognitive empathy $\times$ affective empathy & 0.324 & .197 & .105 & 1.64 & .102 \\
\hline Sex $\times$ cognitive empathy & 0.063 & .357 & .014 & 0.18 & .860 \\
\hline Sex $\times$ affective empathy & 0.504 & .356 & .113 & 1.42 & .158 \\
\hline \multicolumn{6}{|l|}{ Desire sociosexuality } \\
\hline Intercept & 4.584 & .169 & & 27.18 & .001 \\
\hline Sex & -2.146 & .261 & -.484 & -8.23 & .001 \\
\hline Cognitive empathy & 0.357 & .168 & .163 & 2.13 & .034 \\
\hline Affective empathy & 0.004 & .167 & .002 & 0.03 & .979 \\
\hline Cognitive empathy $\times$ affective empathy & 0.280 & .146 & .114 & 1.91 & .057 \\
\hline Sex $\times$ cognitive empathy & -0.267 & .266 & -.076 & -1.01 & .316 \\
\hline Sex $\times$ affective empathy & -0.074 & .266 & -.021 & -0.28 & .781 \\
\hline
\end{tabular}

Bold indicates significance at $p>.05$

Behavior $r^{2}=.043$; attitude $r^{2}=.158$; desire $r^{2}=.261$; for sex, male $=0$ and female $=1$ in efficiently finding like-minded partners, since mating strategies vary in the opposite sex and not all individuals may be interested in short-term mating encounters. Arguably, cognitive empathy would be useful in the establishment of any type of social relationship - including long-term mating relationships. However, in the kinds of extended encounters more typically associated long-term relationships, the effect of rapid assessment of emotional mind states may be less salient-in long-term situations, an individual would have access to a much larger body of direct and indirect information on which they can draw to help them assess another's mind state.

The finding that affective empathy was also related to mating strategy, though only when cognitive empathy ability was also high, goes partly against initial predictions that affective empathy may be unrelated, or even negatively related, to shortterm mating. It has been suggested that if one mating partner develops strong feelings of attachment, the automatic reciprocation of those feelings in the other partner may be deleterious to the pursuit of alterative mating opportunities. Furthermore, since improved general empathy abilities appear to be related to long-term relationship survival, low empathy abilities may in turn be expected to be associated with poor long-term relationship survival and thus de facto short-term mating outcomes. The findings presented here suggest that neither of these explanations may be pertinent to the case of shortterm mating orientation. Affective empathy may be equally useful in building rapport between two individuals by quickly and intuitively communication information about feelings arising in one potential partner, thus increasing rapport and easing effective social interaction. The fact that affective and cognitive empathy show an interaction effect with short-term mating suggests that when combined, these two types of empathy may be particularly effective at facilitation the kind of rapid social connections required to successful engage in a short-term mating strategy.

When the sub-components of short-term mating strategy were examined alongside the two types of empathy, only the desire component trended towards showing significant interaction effects between cognitive/affective empathy and mating strategy. This suggests that it may be sexual desire which is driving the relationship between empathy and short-term mating. If individuals high in affective empathy are more likely to 
mirror any positive emotions arising from a sexual encounter, it would make sense that they would be more motivated to pursue more such mating encounters, and combined with high cognitive empathy abilities, they may be better placed to succeed in establishing such mating encounters.

It is possible that the assessment of "affective" empathy used here, which is typically used as a more "general" empathy measure, may just be capturing another aspect of cognitive empathy. However, using factor analysis, past research suggests not only that this scale reliably measures an affective component of empathy but that this scale is particularly sensitive to the affective aspect of empathy (Muncer and Ling 2006). Furthermore, the fact that the cognitive and affective empathy measures used here have little direct interrelationship with each other (i.e., no significant correlation) suggests at face value that they are measuring different facets of empathy ability. Furthermore, the exploratory and cross-sectional nature of this study makes it as yet impossible to infer any kind of causality between empathy and mating strategies. It may be that differing empathy abilities allow for and motivate individuals to pursue one type of strategy over another. It may also be that individuals are forced to adopt a particular mating strategy based on the limitations set by their empathy abilities-i.e., an inability to form and maintain long-term relationships may lead to partaking in short-term relationships only. Differing levels of empathy may not be motivating individuals to pursue one strategy over another, but this differentiation in empathy may make the pursuit of certain mating strategies easier to execute and thus more desirable. Alternatively, it may also be that experience with a large number of short-term relationships improves cognitive empathy abilities over time through the practice effects of having to interpret social cues with multiple individuals. These initial findings leave considerable scope to be explored in future studies, which may be better placed to disentangle causality issues. For example, it may be possible to experimentally manipulate an experience of affective empathy to see if it has an effect on the desire for short-term mating encounters. Alternatively individuals can be primed with shortterm mating cues to see if either their self-reported or experimentally assessed empathy abilities are affected.

Empathy is an important component of general sociality (Roberts and Strayer 2013), i.e., the ability to form and maintain various social relationships. Promiscuous (short term) mating necessitates the successful instigation of social interactions with multiple individuals - and the current study suggests that both affective and cognitive empathy abilities may be related to such mating behaviors.

\section{Acknowledgments The author is supported by an EU grant 295663 to Robin I.M. Dunbar.}

Conflict of interest The author declares that he has no competing interests

\section{References}

Acevedo, B. P., \& Aron, A. (2012). Neural correlates of marital satisfaction and well-being: reward, empathy, and affect. Clinical Neuropsychiatry, 9(1), 20-31. Retrieved from http://www. clinicalneuropsychiatry.org/.

Al-Shawaf, L., Lewis, D. M. G., \& Buss, D. M. (2014). Mating strategy and disgust. Evolution and Human Behavior. doi:10.1016/j. evolhumbehav.2014.11.003.

Andersson, M. B. (1994). Sexual selection. Princeton University Press.

Bailey, J. M., Kirk, K. M., Zhu, G., Dunne, M. P., \& Martin, N. G. (2000). Do individual differences in sociosexuality represent genetic or environmentally contingent strategies? Evidence from the Australian twin registry. Journal of Personality and Social Psychology, 78(3), 537-545. doi:10.1037/0022-3514.78.3.537.

Barnett, M. A., \& Thompson, S. (1985). The role of perspective taking and empathy in children's Machiavellianism, prosocial behavior, and motive for helping. The Journal of Genetic Psychology. doi: 10.1080/00221325.1985.9914459.

Baron-Cohen, S., Leslie, A. M., \& Frith, U. (1985). Does the autistic child have a "theory of mind"? Cognition, 21(1), 37-46. doi:10.1016/ 0010-0277(85)90022-8.

Baron-Cohen, S., Jolliffe, T., Mortimore, C., \& Robertson, M. (1997). Another advanced test of theory of mind: evidence from very high functioning adults with autism or Asperger syndrome. Journal of Child Psychology and Psychiatry, and Allied Disciplines, 38(7), 813-822. doi:10.1111/j.1469-7610.1997.tb01599.x.

Baron-Cohen, S., Wheelwright, S., Hill, J., Raste, Y., \& Plumb, I. (2001). The "Reading the Mind in the Eyes" test revised version: a study with normal adults, and adults with Asperger syndrome or highfunctioning autism. Journal of Child Psychology and Psychiatry, 42(2), 241-251. doi:10.1111/1469-7610.00715.

Batson, C. D. (2009). These things called empathy: eight related but distinct phenomenon. In J. Decety \& W. Ickes (Eds.), The social neuroscience of empathy (pp. 16-28). Cambridge, MA: MIT Press.

Blair, R. J. R. (2005). Responding to the emotions of others: dissociating forms of empathy through the study of typical and psychiatric populations. Consciousness and Cognition, 14(4), 698-718. doi:10. 1016/j.concog.2005.06.004.

Blair, R. J. R. (2006). The emergence of psychopathy: implications for the neuropsychological approach to developmental disorders. Cognition, 101, 414-442. doi:10.1016/j.cognition.2006.04.005.

Bleske-Rechek, A., VandenHeuvel, B., \& Vander Wyst, M. (2009). Age variation in mating strategies and mate preferences: beliefs versus reality. Evolutionary Psychology, 7(2), 179-205.

Boettcher, R. E. (1978). Interspousal empathy, marital satisfaction, and marriage counseling. Journal of Social Service Research, 1(1), 105113. doi:10.1300/J079v01n01 08.

Busby, D. M., \& Gardner, B. C. (2008). How do I analyze thee? Let me count the ways: considering empathy in couple relationships using self and partner ratings. Family Process, 47(2), 229-242. doi:10. 1111/j.1545-5300.2008.00250.x.

Buss, D. M., \& Schmitt, D. P. (1993). Sexual strategies theory: an evolutionary perspective on human mating. Psychological Review, 100(2), 204-232. doi:10.1037/0033-295X.100.2.204.

Clutton-Brock, T. H. (1989). Review lecture: mammalian mating systems. Proceedings of the Royal Society B: Biological Sciences, 236(1285), 339-372. doi:10.1098/rspb.1989.0027.

Cramer, D., \& Jowett, S. (2010). Perceived empathy, accurate empathy and relationship satisfaction in heterosexual couples. Journal of Social and Personal Relationships, 27(3), 327-349. doi:10.1177/ 0265407509348384.

Davis, M. H., \& Oathout, H. A. (1987). Maintenance of satisfaction in romantic relationships: empathy and relational competence. Journal 
of Personality and Social Psychology, 53(2), 397-410. doi:10. 1037//0022-3514.53.2.397.

Frith, U., Morton, J., \& Leslie, A. M. (1991). The cognitive basis of a biological disorder: autism. Trends in Neurosciences, 14(10), 433438.

Gangestad, S. W., \& Simpson, J. A. (2000). The evolution of human mating: trade-offs and strategic pluralism. Behavioral and Brain Sciences, 23, 573-644. doi:10.1017/S0140525X0000337X.

Geary, D. C. (2000). Evolution and proximate expression of human paternal investment. Psychological Bulletin, 126(1), 55-77. doi:10. 1037/0033-2909.126.1.55.

Hare, R. D. (1980). A research scale for the assessment of psychopathy in criminal populations. Personality and Individual Differences, 1(2), 111-119. doi:10.1016/0191-8869(80)90028-8.

Haselton, M. G., \& Buss, D. M. (2001). The affective shift hypothesis: the functions of emotional changes following sexual intercourse. Personal Relationships, 8(4), 357-369. doi:10.1111/j.1475-6811. 2001.tb00045.x.

Henry, J. D., Bailey, P. E., \& Rendell, P. G. (2008). Empathy, social functioning and schizotypy. Psychiatry Research, 160(1), 15-22. doi:10.1016/j.psychres.2007.04.014.

Howell, E. C., Etchells, P. J., \& Penton-Voak, I. S. (2012). The sexual overperception bias is associated with sociosexuality. Personality and Individual Differences, 53(8), 1012-1016. doi:10.1016/j.paid. 2012.07.024.

Jankowiak, W. R., \& Fischer, E. F. (1992). A cross-cultural perspective on romantic love. Ethnology, 31(2), 149-155. doi:10.2307/3773618.

Jonason, P. K., \& Buss, D. M. (2012). Avoiding entangling commitments: tactics for implementing a short-term mating strategy. Personality and Individual Differences, 52, 606-610. doi:10.1016/j.paid.2011. 12.015 .

Lawrence, E. J., Shaw, P., Baker, D., Baron-Cohen, S., \& David, A. S. (2004). Measuring empathy: reliability and validity of the Empathy Quotient. Psychological Medicine, 34(5), 911-919. doi:10.1017/ S0033291703001624.

Levesque, C., Lafontaine, M.-F., Caron, A., Flesch, J. L., \& Bjornson, S. (2014). Dyadic empathy, dyadic coping, and relationship satisfaction: a dyadic model. Europe's Journal of Psychology, 10(1), 118134. doi:10.5964/ejop.v10i1.697.

Lippa, R. A. (2009). Sex differences in sex drive, sociosexuality, and height across 53 nations: testing evolutionary and social structural theories. Archives of Sexual Behavior, 38(5), 631-651. doi:10.1007/ s10508-007-9242-8.

Long, E. C. J., Angera, J. J., Carter, S. J., Nakamoto, M., \& Kalso, M. (1999). Understanding the one you love: a longitudinal assessment of an empathy training program for couples in romantic relationships. Family Relations, 48(3), 235-242. doi:10.2307/585632.

Muncer, S. J., \& Ling, J. (2006). Personality and individual differencespsychometric analysis of the Empathy Quotient (EQ) scale. Personality and Individual Differences, 40, 1111-1119. doi:10. 1016/j.paid.2005.09.020.

Paulhus, D. L., \& Williams, K. M. (2002). The Dark Triad of personality: narcissism, Machiavellianism, and psychopathy. Journal of Research in Personality, 36, 556-563. doi:10.1016/S00926566(02)00505-6.

Penke, L., \& Asendorpf, J. B. (2008). Beyond global sociosexual orientations: a more differentiated look at sociosexuality and its effects on courtship and romantic relationships. Journal of Personality and Social Psychology, 95(5), 1113-1135. doi:10.1037/0022-3514.95. 5.1113.

Perrone-McGovern, K. M., Oliveira-Silva, P., Simon-Dack, S., LefdahlDavis, E., Adams, D., McConnell, J., \& Gonçalves, O. F. (2014). Effects of empathy and conflict resolution strategies on psychophysiological arousal and satisfaction in romantic relationships. Applied Psychophysiology and Biofeedback, 39(1), 19-25. doi:10.1007/ s10484-013-9237-2.
Premack, D., \& Woodruff, G. (1978). Does the chimpanzee have a theory of mind? Behavioral and Brain Sciences, 04, 515-526. doi:10.1017/ S0140525X00076512.

Roberts, W., \& Strayer, J. (2013). Empathy, emotional prosocial behavior expressiveness, and prosocial behavior. Child Development, 67(2), 449-470.

Sacco, D. F., Hugenberg, K., \& Sefcek, J. A. (2009). Sociosexuality and face perception: unrestricted sexual orientation facilitates sensitivity to female facial cues. Personality and Individual Differences, 47(7), 777-782. doi:10.1016/j.paid.2009.06.021.

Saxe, R. (2006). Uniquely human social cognition. Current Opinion in Neurobiology, 16(2), 235-239. doi:10.1016/j.conb.2006.03.001.

Schmitt, D. P. (2005). Sociosexuality from Argentina to Zimbabwe: a 48nation study of sex, culture, and strategies of human mating. Behavioral and Brain Sciences, 28(2), 247-275. doi:10.1017/ S0140525X05000051. discussion 275-311.

Schmitt, D. P. (2008). Big five traits related to short-term mating: from personality to promiscuity across 46 nations. Evolutionary Psychology, 6(2), 246-282.

Schurz, M., Radua, J., Aichhorn, M., Richlan, F., \& Perner, J. (2014). Fractionating theory of mind: a meta-analysis of functional brain imaging studies. Neuroscience and Biobehavioral Reviews, 42, 9 34. doi:10.1016/j.neubiorev.2014.01.009.

Shamay-Tsoory, S. G., Aharon-Peretz, J., \& Perry, D. (2009). Two systems for empathy: a double dissociation between emotional and cognitive empathy in inferior frontal gyrus versus ventromedial prefrontal lesions. Brain, 132(Pt 3), 617-627. doi:10.1093/brain/ awn279.

Shultz, S., \& Dunbar, R. I. M. (2007). The evolution of the social brain: anthropoid primates contrast with other vertebrates. Proceedings of the Royal Society B: Biological Sciences, 274(1624), 2429-2436. doi:10.1098/rspb.2007.0693.

Simpson, J. A., Gangestad, S. W., Bogart, H., Grudrein, D., Hall, M., Hutchins, L., \& Williams, R. (1991). Individual differences in sociosexuality: evidence for convergent and discriminant validity. Journal of Personality, 60(6), 870-883. doi:10.1037/0022-3514. 60.6 .870

Singer, T. (2006). The neuronal basis and ontogeny of empathy and mind reading: review of literature and implications for future research. Neuroscience and Biobehavioral Reviews, 30(6), 855-863. doi:10. 1016/j.neubiorev.2006.06.011.

Smith, D. S., Jones, B. C., \& Allan, K. (2013). Socio-sexuality and episodic memory function in women: further evidence of an adaptive "mating mode". Memory \& Cognition, 41(6), 850-861. doi:10. 3758/s13421-013-0301-1.

Trivers, R. L. (1972). Parental investment and sexual selection. In B. Campbell (Ed.), Sexual selection and the descent of man (pp. 136179). New Brunswick: Aldine.

Van Honk, J., Schutter, D. J., Bos, P. A., Kruijt, A.-W., Lentjes, E. G., \& Baron-Cohen, S. (2011). Testosterone administration impairs cognitive empathy in women depending on second-to-fourth digit ratio. Proceedings of the National Academy of Sciences, 108(8), 34483452. doi:10.1073/pnas.1011891108.

Wai, M., \& Tiliopoulos, N. (2012). The affective and cognitive empathic nature of the dark triad of personality. Personality and Individual Differences, 52(7), 794-799. doi:10.1016/j.paid.2012.01.008.

Wakabayashi, A., Baron-Cohen, S., Wheelwright, S., Goldenfeld, N., Delaney, J., Fine, D., \& Weil, L. (2006). Development of short forms of the Empathy Quotient (EQ-Short) and the Systemizing Quotient (SQ-Short). Personality and Individual Differences, 41(5), 929940. doi:10.1016/j.paid.2006.03.017.

Walter, H. (2012). Social cognitive neuroscience of empathy - concepts, circuits and genes. Emotion Review, 4(1), 9-17. doi:10.1177/ 1754073911421379

Watson, P. J., Grisham, S. O., Trotter, M. V., \& Biderman, M. D. (1984). Narcissism and empathy: validity evidence for the narcissistic 
personality inventory. Journal of Personality Assessment. doi:10. 1207/s15327752jpa4803_12.

Waynforth, D., Delwadia, S., \& Camm, M. (2005). The influence of women's mating strategies on preference for masculine facial architecture. Evolution and Human Behavior, 26(5), 409-416. doi:10. 1016/j.evolhumbehav.2005.03.003.
Wlodarski, R., \& Dunbar, R. I. M. (2014). The effects of romantic love on mentalizing abilities. Review of General Psychology, 18(4), 313321. doi:10.1037/gpr0000020.

Wlodarski, R., Manning, J. T., \& Dunbar, R. I. M. (2015). Stay or stray? Evidence for alternative mating strategy phenotypes in both men and women. Biology Letters, 11(2), 20140977. doi:10.1098/rsbl.2014.0977. 